\title{
Mechanisms for substance use disorders in COVID-19
}

\author{
Zhicheng Lin ${ }^{1}$
}

Received: 10 November 2020 / Revised: 14 January 2021 / Accepted: 25 January 2021 / Published online: 15 February 2021

(c) The Author(s), under exclusive licence to Springer Nature Limited 2021

\section{To the Editor:}

I read with interest Wang and colleagues' epidemiological findings that substance use disorders (SUDs), such as opioid (OUD), tobacco (TUD), alcohol (AUD) and cocaine (CUD) use disorders, are correlated with COVID-19 [1]. They found that OUD conferred the greatest risk for COVID-19, followed by TUD and AUD, all in large effect sizes (adjusted odds ratio $(\mathrm{AOR})=10.2$ for OUD, 8.2 for TUD and 7.8 for AUD). They also found the risk was greater in African Americans than in European Americans. I was assuming that the authors were focused on symptomatic COVID-19 which represents developed forms of this disease. We might interpret these impressive findings with vulnerability for the disease development (from infection to symptom appearing) as well as progression (from symptom appearing to severe forms such as Intensive Care Unit (ICU) necessity and death). By searching the literature for SUDs effects on immune system and organ injury, and genetics, the vulnerability can be understood mechanistically.

SUDs may damage the immune system [2-4]. COVID19 is an infectious disease so that its development and progression are regulated largely by the immune system. This infectious disease may progress to severe forms in patients with SUDs likely attributable to weakened immunity as a result of SUDs.

On the other hand, clinical investigations observed that SUDs may upregulate expression of the receptor ACE2 for SARS-CoV-2, which is the coronavirus causing COVID19. For example, patients with TUD carried elevated ACE2

Supplementary information The online version contains supplementary material available at https://doi.org/10.1038/s41380021-01041-0.

Zhicheng Lin

zlin@mclean.harvard.edu

1 Laboratory of Psychiatric Neurogenomics, McLean Hospital, Harvard Medical School, Belmont, MA, USA expressions in their airway [5, 6]. This line of evidence suggests that patients with SUDs are vulnerable to the infection. ACE2 happens to be a receptor for morphine and dynorphin too but it remains yet unclear whether this function involves COVID-19.

Hence, the disease development may target those with SUDs. If this is true, patients with COVID-19, especially those with severe forms, may carry known genetic risks for SUDs. To test this molecular hypothesis, literature was searched and obtained genetic evidence is summarized as a SUDs-related 60-gene network in Supplementary Table (see Ref. 7 for genetic databases used).

A recent genome-wide association study (GWAS) identified the ubiquitously expressed transcription factor LZTFL1 as the most significant genetic risk for severe COVID-19 in Europeans [7]. Interestingly, LZTFL1 has been found by Gelernter and colleagues as a genetic risk also for modulating methadone dosing in European American, not in African American, patients with OUD, based on six markers across the gene $\left(\beta=-0.23 \sim-0.35, P=4.1 \sim 1.6 \times 10^{-5}\right)[8]$, implying its population-dependent genetic role in opioid signaling. Furthermore, LZTFL1 displayed a protective effect against COVID-19 severity $\left(\mathrm{AOR}_{\text {meta }}=0.56 \sim 0.47\right)$ in the European patients, consistent with the epidemiological observation of less exacerbating effect of SUDs in European Americans than African Americans. Of the note, the six methadone-dosing markers were not associated with COVID-19 severity, suggesting that two different functional haplotypes be involved. The regulatory mechanisms of LZTFL1 thus warrant future investigation.

As more consistency, seven others of the sixty genes are also associated with severe COVID-19 in the GWAS, including PDYN, BDNF, ENTPD6, ADHIC, HEY1, PLAGL1, and HIVEP2 (Supplementary Table). A half of the eight significant associations are in genes encoding neuronal transcription factors (TFs), which was a 4-fold enrichment comparing to non-TF associations. HEYl showed the next most significant association. This gene is a TF known for AUD/TUD-related SLC6A3, another gene with nominal significant $P_{\text {meta }}$ values here. Comparing to $A D H 1 B, A D H 1 C$ 


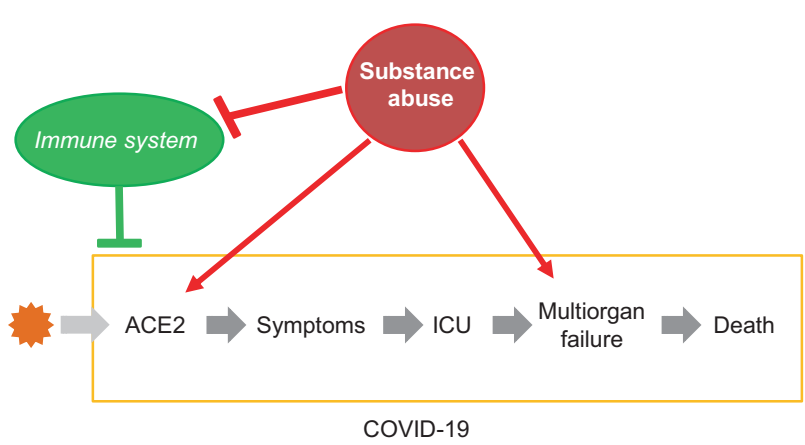

Fig. 1 Triple action of substance abuse in COVID-19. Brown, the coronavirus SARS-CoV-2. In addition, AUD might cause behavioral disinhibition for increased exposure to infection.

$\left(\mathrm{AOR}_{\text {meta }}=0.4\right)$ showed greater associations in terms of both significance and effect size. AUD has been implicated in multiple organ damage [9] and is well positioned to contribute to multiorgan failure which is a common cause of COVID-19-related mortality [10]. Based on an approximate estimation, this network has $>85 \%$ of $P_{\text {meta }}$ values as $<0.05$, which is a 17-fold enrichment comparing to the whole GWAS data $(\sim 5.2 \%)$. These data suggest that this SUDsrelated genetics is well implicated in patients with severe COVID-19, supporting the hypothesis that patients with COVID-19, especially those with severe forms, may carry known genetic risks for SUDs; that is, COVID-19 development and progression target those with SUDs. Clinically, these shared genetic risks may help to identify individuals at high risk for the comorbidity development.

To sum up (Fig. 1), SUDs can exacerbate SARS-CoV-2 infection in three ways, that is, facilitating the viral entry, impairing the immune system, and injuring other organs such as heart, lung, liver and kidney, which also highlights the systemic toxicity of abused substances. For the moment, this may help explain how COVID-19 targets patients with SUDs in a large effect size manner.

Acknowledgements This work is supported by U.S. National Institute on Drug Abuse grant (NIDA) DA021409, and National Institute on Alcohol Abuse and Alcoholism grant (NIAAA) AA026663.
Funding The funder of the work had no role in conception, data retrieving or interpretation, and writing. I had full access to all the data mentioned and final responsibility for the decision to submit for publication.

\section{Compliance with ethical standards}

Conflict of interest The author declares that he has no conflict of interest.

Publisher's note Springer Nature remains neutral with regard to jurisdictional claims in published maps and institutional affiliations.

\section{References}

1. Wang QQ, Kaelber DC, Xu R, Volkow ND. COVID-19 risk and outcomes in patients with substance use disorders: analyses from electronic health records in the United States. Mol Psych. 2021;26:30-9.

2. Cook RT. Alcohol abuse, alcoholism, and damage to the immune system-a review. Alcohol, Clin Exp Res. 1998;22:1927-42.

3. Roy S, Loh HH. Effects of opioids on the immune system. Neurochem Res. 1996;21:1375-86.

4. Stampfli MR, Anderson GP. How cigarette smoke skews immune responses to promote infection, lung disease and cancer. Nat Rev Immunol. 2009;9:377-84.

5. Leung JM, Yang CX, Tam A, Shaipanich T, Hackett TL, Singhera GK, et al. ACE-2 expression in the small airway epithelia of smokers and COPD patients: implications for COVID-19. Eur Resp J. 2020;55:1-5.

6. Leung JM, Yang CX, Sin DD. COVID-19 and nicotine as a mediator of ACE-2. Eur Resp J. 2020;55:2001261. https://doi.org/ 10.1183/13993003.01261-2020.

7. Ellinghaus D, Degenhardt F, Bujanda L, Buti M, Albillos A, Invernizzi $\mathrm{P}$, et al. Genomewide association study of severe covid19 with respiratory failure. N Engl J Med. 2020;383:1522-34.

8. Smith AH, Jensen KP, Li J, Nunez Y, Farrer LA, Hakonarson H, et al. Genome-wide association study of therapeutic opioid dosing identifies a novel locus upstream of OPRM1. Mol Psychiatry. 2017;22:346-52.

9. Osna NA, Kharbanda KK. Multi-organ alcohol-related damage: mechanisms and treatment. Biomolecules. 2016;6:20. https://doi. org/10.3390/biom6020020.

10. Gupta A, Madhavan MV, Sehgal K, Nair N, Mahajan S, Sehrawat TS, et al. Extrapulmonary manifestations of COVID-19. Nat Med. 2020;26:1017-32. 